\title{
CURSOR CONTROL DEVICE CHARACTERISTICS
}

\author{
James G. Phillips' and Thomas J. Triggs \\ 'Psychology Department, Monash University, Clayton VIC Australia, \\ email: James.G.Phillips@sci.monash.edu.au
}

\begin{abstract}
An analysis of cursor positioning may provide guidelines for improvements in cursor control. Four experiments addressed efficiency of cursor control devices (Mouse, Digitising Pen, Accupoint, Trackball). Participants moved a cursor leftwards, upwards or rightwards, positioning it in large or small targets situated in near or far space on the computer screen. Cursor coordinates were sampled every $5 \mathrm{~ms}$. The number of submovements and the proportion of time spent in deceleration were analysed. Participants could not plan movements controlled by an Accupoint. Cursor trajectories were more variable in near space for detachable manipulanda due to potential cartesian coordinate system incompatibilities.
\end{abstract}

KEY WORDS:

Cursor, Positioning, Kinematics, Mouse, Accupoint, Trackball, Pen

\section{INTRODUCTION}

Cursor control devices differ in their intuitive appeal, their directness, and the demands they make on space and posture (Baber, 1997). Although the Mouse is commonly used in personal computers, recent developments in laptop computers and personal organisers have led to an increase in the use of alternative cursor control devices, and a reemergence of choice (Dix, Finlay, Abowd, \& Beale, 1998), with concerns that good ergonomic practice may be sacrificed in some instances for compactness. There is thus a need to characterise the properties of cursor control devices when positioning cursors upon computer screens. A better understanding of the nature of such positioning movements may provide informed choice as to the appropriate cursor control device for a specific application, and/or assist in the development of real-time algorithms that can predict movement endpoints.

Pointing devices are typically assumed to be adequately described by Fitts' (1954) law, which describes the rate of gain of movement precision with longer movement durations. However, Fitts' law is a simplification, rather than an explanation of the underlying mechanisms (MacKenzie, 1992). Indeed, longer durations for movements of greater precision may be due to a greater need: 1) to overcome the variation in the initial impulse; 2) for terminal visual guidance; 3 ) for successive corrective responses; 4 ) to overcome incompatiblities of display and control (Wickens, Gordon, \& Liu, 1998). And while Fitts' law holds for a variety of tasks (e.g. Langolf, Chaffin, \& Foulke, 1976), it does not apply to less precise movements (Gan \& Hoffman, 1988). Nor does Fitts' law provide guidelines for any interventions to improve positioning movements (MacKenzie, 1992), because it does not address the underlying mechanisms responsible for the coordination of movement (MacKenzie, 1992), thereby overlooking the rich potential associated with current technology. Since cursor coordinates are monitored in real time there is the potential to reduce the duration of positioning movements if the intended endpoint can be predicted effectively (Murata, 1995).

There has only been a limited application of kinematic techniques to address the underlying nature of cursor positioning movements using a restricted number of cursor control devices. Walker, Meyer and Smelcer (1993) examined the number of submovements required when a Mouse was used to move a cursor over different distances to rectangular targets of varying horizontal and vertical dimensions. Walker et al. found the number of submovements increased when movements were made to targets requiring greater precision. Subsequently, Douglas and Mithal (1997) addressed the efficacy of Mouse and isometric joystick (Trackpoint). They reported that the Trackpoint was less efficient, there being an appreciable variability in the trajectories produced by this device. They attributed the poorer performance to high frequency noise within the device (removable by filtering), and oscillations due to operator tremor. And while Douglas and Mithal thought tremor was a contributing factor, there was little evidence for the rhythmic motions characteristic of tremor, indicating other factors are likely. Indeed increased variability in cursor trajectories might reflect an increased need for visually based corrective responses. Unfortunately, the proportion of time spent in terminal guidance was not considered, and so it was difficult to address such issues. However, it may be possible to answer these issues by a judicious comparison of cursor control devices.

There are a number of cursor control devices available (Dix et al., 1998), which vary in their efficacy (MacKenzie, 1992). Differences in their efficacy may reflect their mode of operation. Devices such as isometric joysticks and Trackballs afford fewer cues to their operation, while the Mouse and Digitising Pen are somewhat more intuitive and direct (Dix et al., 1998). In the present paper we summarise 4 of our studies in which we used kinematic techniques to characterise properties of cursor control devices. We report the impact of movement distance and target size upon cursor trajectories controlled by Mouse, Digitising Pen, Accupoint, and Trackball. Since direction of motion has been observed to affect positioning time (Tränkle \& Deutschmann, 1991), we compared leftwards diagonal, vertical and rightwards diagonal movements. As geometrical factors have had an impact upon cursor positioning times (Walker et al., 1993), 
circular targets were used to control target approach angle. Kinematic analysis of cursor positioning movements can document: 1) the variability of the accelerative phase of movement; 2) the duration of the decelerative phase of movement; 3) the numbers of submovements and accelerative/decelerative impulses. This analysis should provide insights into relative strengths and weaknesses associated with each cursor control device.

\section{METHOD}

Twelve right handed adults participated in each experiment. The experiments were performed using: 1) Microsoft Mouse and a 486PC; 2). a Digitising Pen, WACOM SD420 graphics tablet and 486PC; 3) an Accupoint on a Toshiba pentium laptop computer; 4) the Trackball on a MTAC 486 laptop computer. In each case the computer sampled cursor coordinates every $5 \mathrm{~ms}$. Participants sat in the midline of the computer, viewing the screen from a distance of $40 \mathrm{~cm}$. Participants used the cursor control device to move a cursor from a home position at the middle of the bottom of the screen, to a target of either 6 or $12 \mathrm{~mm}$ diameter, that could be situated $5.35 \mathrm{~cm}$ or $10.7 \mathrm{~cm}$ away. The target could appear in the vertical midline of the screen, directly above the home target, or could be situated 30 degrees to the right or 30 degrees to the left of the midline. These combinations of target sizes (big, small), distances (near, far) and directions (leftwards, middle, rightwards) produced a $2 \times 2 \times 3$ design.

Participants were instructed to position the cursor in the home position upon the computer screen. A target (either big or small) was then presented in one of six possible randomly determined locations. Trials terminated after the cursor entered the target. After a block of 24 practice trials, there were 5 blocks of 24 experimental trials, providing 10 trials for each combination of target size, distance, and direction. Kinematic indices were calculated for each trial, and then averaged for each condition.

Kinematic features were determined along the main axis of movement (i.e. in the direction of the target). High frequency noise affecting the coordinates generated by the devices were filtered out. The coordinates of the resulting displacement function were then differentiated twice to produce velocity and acceleration functions. Reaction time was defined as the duration of time from initial button click to onset of cursor movement. Movement time was defined as the interval of time from onset of cursor movement to the point in time at which maximum displacement occurred. The point of maximum extent along the main axis of movement was used to determine the extent to which people overshoot the target. The proportion of total movement duration spent in deceleration was determined using the point at which peak velocity occurred: the period of time from peak velocity to maximum displacement was divided by total movement duration. The angular error of aiming was determined from an angle defined by lines joining: the centre of the target and the point at which movement started; and the point at which movement started and the point at which peak velocity occurred. The number of submovements was determined by counting zero-crossings in the velocity function, and the number of cycles of acceleration and deceleration was determined by counting zero-crossings in the acceleration function. These kinematic indices were then subjected to separate $2 \times 2 \times 3$ repeated measures analysis of variance.

\section{RESULTS}

Findings are summarised in Table 1. The flatter slope for Fitts' law for Accupoint and Trackball indicated that operators require movements of longer duration to place the cursor upon the computer screen with equivalent precision to that of Mouse and Digitising Pen. Some additional insights may be obtained from response latencies. Accupoint and Trackball had longer response latencies indicating that fewer cues are afforded as to their operation. The Accupoint showed no systematic changes in Reaction Time as a function of task difficulty, implying there are problems planning cursor movements with this device. This may be reflected in the predictability of the cursor trajectory. Mouse and Pen require fewer accelerative/decelerative impulses per second than Accupoint or Trackball. The Trackball is particularly notable for the large number of submovements it requires. This jerkiness means that cursor trajectories are less stable and predictable for Accupoint and Trackball.

The percentage of movement time spent in deceleration, provides an index as to the amount of terminal guidance required with each device. For most of these cursor control devices, the values are greater than those associated with ordinary pointing movements (e.g. 55\%-65\%), this indicates a greater reliance upon visual feedback to execute these movements. The Accupoint is the exception here, but this may actually arise from the prolonged periods of time spent in acceleration for this cursor control device. As a lump in the middle of the keyboard, the Accupoint affords few cues to its operation, and this would appear to lead to slower initiation of responses and greater uncertainty during the initial accelerative and terminal guidance phases of positioning movements. The greater degree of overshoot associated with the Accupoint emphasises the problems people have with this cursor control device. 


\begin{tabular}{|c|c|c|c|c|}
\hline & MOUSE & PEN & ACCUPOINT & TRACKBALL \\
\hline Slope Fitts' law & 10.8 bits/s & 6.94 bits/s & 5.72 bits/s & 3.30 bits/s \\
\hline Reaction Time & 239.7 & 284.8 & 460.6 & 588.9 \\
ms & $(4.8)$ & $(4.7)$ & $(8.0)$ & $(21.4)$ \\
\hline Movement & 643.2 & 646.8 & 833.9 & 974.1 \\
Time ms & $(10.7)$ & $(16.8)$ & $(24.5)$ & $(39.6)$ \\
\hline Overshoot mm & 2.67 & 0.67 & 27.8 & 9.07 \\
& $(0.41)$ & $(0.31)$ & $(2.0)$ & $(0.61)$ \\
\hline No. of & 2.20 & 2.40 & 2.09 & 14.58 \\
Submovements & $(0.05)$ & $(0.06)$ & $(0.05)$ & $(0.46)$ \\
\hline Force & 7.85 & 6.29 & 10.80 & 11.90 \\
Incfficiency & $(0.10)$ & $(0.08)$ & $(0.15)$ & $(0.07)$ \\
\hline Percent Time in & 71.0 & 81.4 & 56.1 & 73.2 \\
Deceleration & $(0.52)$ & $(0.37)$ & $(1.18)$ & $(0.93)$ \\
\hline Angular Error ${ }^{2}$ & 4.3 & 4.5 & 4.9 & 5.74 \\
& $(0.25)$ & $(0.24)$ & $(0.54)$ & $(0.31)$ \\
\hline
\end{tabular}

${ }^{1}$ Number of accelerative/decelerative impulses per second

${ }^{2}$ Degrees error at peak velocity from the direct path to the target

\section{Table 1. Relative efficacy of cursor control devices}

Each of these cursor control devices tends to show the expected changes in performance as greater movement precision is required, that is greater movement durations for more precise (i.e. smaller targets) movements. However, there were some unexpected effects. Cursor positioning times tended to be faster, but less accurate to targets on the left of the screen, and there were problems with diagonal movements, which we attribute to additional problems dealing with two coordinate axes (both $\mathrm{X}$ and $\mathrm{Y}$ ) instead of one ( $\mathrm{Y}$ ). In addition, while we expected cursor positioning to be easier for near targets, the Mouse and Digitising Pen exhibited the reverse for two kinematic indices.

As may be seen in Table 2, there were increased cycles of acceleration and deceleration per second for near targets for the Mouse and Pen. In addition, there was increased error associated with the point at which peak velocity was attained. There was a greater angular error of aiming for the near target for the Mouse and Pen. In contrast, for the Accupoint and Trackball the greater angular error was associated with the further targets. This means that the cursor trajectories were less predictable in near space for the Mouse, but that cursor trajectories were less predictable in far space for the Accupoint. There are clearly additional factors influencing cursor control using Mouse and Pen.

\begin{tabular}{|c|c|c|c|c|}
\hline & MOUSE & TABLET & ACCUPOINT & TRACKBALL \\
\hline $\begin{array}{c}\text { FORCE } \\
\text { INEFFICIENCY }\end{array}$ & $\begin{array}{c}\mathrm{F}(1,11)=35.0, \\
\mathrm{p}<0.001\end{array}$ & $\begin{array}{c}\mathrm{F}(1,11)=140.9, \\
\mathrm{p}<0.001\end{array}$ & $\begin{array}{c}\mathrm{F}(1,11)=12.3, \\
\mathrm{p}<0.005\end{array}$ & $\begin{array}{c}\mathrm{F}(1,11)=0.9, \\
\mathrm{p}>0.05\end{array}$ \\
\hline near & 8.26 & 6.85 & 10.18 & 11.86 \\
\hline far & 7.44 & 5.72 & 11.45 & 11.96 \\
\hline $\begin{array}{c}\text { ANGULAR }_{\text {ERROR }^{2}} \\
\text { near }\end{array}$ & $\begin{array}{c}\mathrm{F}(1,11)=5.28, \\
\mathrm{p}<0.05\end{array}$ & $\begin{array}{c}\mathrm{F}(1,11)=30.6, \\
\mathrm{p}<0.001\end{array}$ & $\begin{array}{c}\mathrm{F}(1,11)=5.94, \\
\mathrm{p}<0.05\end{array}$ & $\begin{array}{c}\mathrm{F}(1,11)=3.93, \\
\mathrm{p}<0.07\end{array}$ \\
\hline far & 4.83 & 4.94 & 3.67 & 5.46 \\
\hline
\end{tabular}

${ }^{1}$ Number of accelerative/decelerative impulses per second

${ }^{2}$ Degrees error at peak velocity from the direct path to the target

Table 2. Differential effects of movement amplitude

\section{DISCUSSION}

The increasing variety of alternative cursor control devices available for laptop or desktop computers necessitates further considerations of the grounds upon which choice can be made between devices. Baber (1997) distinguishes between cursor control devices on the basis of their learnability, effectiveness (speed, accuracy), acceptability and flexibility. To some extent, the effectiveness of a cursor control device can be indicated by Fitts' law, with the efficiency of cursor control impacting upon the acceptability of the device as far as sustained use in relatively fixed postures is concerned. Nevertheless, Fitts' law does not address the underlying effectors or the associated trajectories during cursor control, and this can be a problem when considering possible improvements to hardware or enhancements to software (MacKenzie, 
1992). In this regard, our kinematic analysis of cursor trajectories produced with a variety of cursor control devices provides some insights into the characteristics of the controllers and the predictability of the trajectories they produce.

Fitts' law provides a rate of gain of movement precision for movements of longer duration, and this can be used to compare the four cursor control devices in this study. In this regard the Mouse is superior to Digitising Pen, Accupoint and Trackball in keeping with reviews by MacKenzie (1992). The values for the slopes of Fitts' law are also in keeping with those of previous studies, which is pleasing given the degree of discrepancy observed between studies (Baber, 1997). And while Fitts' law is reported to hold for a variety of effectors over a range of tasks, the slope also varies as a function of the muscle groupings employed (Langolf, Chaffin, \& Foulke, 1976).

A number of factors may contribute to the effectiveness of cursor control devices. Mouse, Digitising Pen, Accupoint and Trackball vary in terms of the grip (palm, finger, finger-tip) and joint combinations (forearm/wrist, finger/wrist, finger) employed during their operation (Baber, 1997). And as the different cursor controllers require different amounts of reaching and grasping, they are liable to employ different neurophysiological systems (Goodale, Jakobson, \& Servos, 1996). Goodale et al. (1996) suggested that a dorsal system involving posterior parietal cortex was more responsible for on-line control of goal directed action, while a ventral system involving inferotemporal cortex was more devoted to object properties. The distinction is important, as it implies the dorsal system is better suited to the processing of distance and the control of reaching movements, while the ventral system is better equipped to deal with orientation and the control of grasping movements. As detachable manipulanda, the Mouse and Digitising Pen can be picked up in a variety of orientations, many of which do not match the coordinate system of the screen. These variations in orientation lead to potential screen/manipulandum cartesian coordinate system incompatibilities, in which operators need to correct for variations in the orientation of these cursor control devices relative to the screen. We suspect the operator initially needs to engage in on-line corrections to establish the mappings between screen and manipulandum, and that this would be a continuing problem, since the standard 2:1 gain means that slight variations in orientation are magnified upon the computer screen. In this regard, relationships of controller to the screen, and the coding of distance varied, with the Accupoint and Trackball having a fixed relationship to the screen in laptops, but at the same time affording a less direct means of coding the distances to be moved. Such factors would appear to contribute to the observed differences in the slope of Fitts' law for these cursor control devices. The present data suggest that variations in biomechanical and neurophysiological systems manifest as greater uncertainty in the initial cursor trajectories (as variations in orientation are mapped in) or greater irregularity during the entire cursor trajectory (as distance is mapped out). The emergence of hybrid cursor control devices comprising mouse and cursor wheel or trackball may indicate manufacturers are sensitive to these issues. An alternative solution to these problems might involve the use of touchsensitive screens or display tablets, but parallax errors and expense currently render these options unsuitable.

While cumulative trauma disorder is more commonly linked to keyboard use (Harms-Ringdahl \& Schüldt, 1990), it does occur with mouse use (Fogleman \& Brogmus, 1995), particularly when mouse placement promotes inappropriate postures (English \& Andre, 1999; Fogleman \& Brogmus, 1995). Some therapeutic interventions have replaced a cursor control device responsible for cumulative trauma disorder with alternative cursor control devices (Hittle, 1999). However, there are reasons to suspect some of the replacement devices (e.g. joystick, trackball) may not be as effective, or be more appropriate for certain specific applications, and indeed be just as likely to lead to problems if used in sustained awkward postures (Hittle, 1999). From our data, a principle advantage of joystick and trackball would seem to be the relatively fixed relationship between controller and screen in laptops, which might mean a reduced need to adjust for differences in orientation between screen and controller in the early part of the cursor trajectory. Nevertheless, while chosen for compactness, these devices are poorer for full screen movements and other approaches may be desirable.

Attempts have been made to improve cursor positioning times by means of software interventions which focus upon the accelerative or decelerative phase of movement. For example, Murata (1995) attempted to predict cursor endpoint from its initial movement trajectory. Conversely, Kabbash and Buxton (1995) sought to improve cursor positioning by using a larger area cursor which captured icons and reduced the amounts of terminal guidance required. This study documents the proportions of time the cursor spends in acceleration or deceleration. Most cursor control devices produce cursor trajectories with considerable amounts of terminal guidance, and where this is not the case (Accupoint) the cursor trajectory itself may not be as predictable. Our data implies a potential uncertainty with the initial cursor trajectory would make an area cursor (Kabbash \& Buxton, 1995) more effective for Mouse and Digitising Pen, while a predictive algorithm based upon the initial cursor trajectory could be more beneficial for Accupoint and Trackball (Murata, 1995). Unfortunately the greater instability of cursor trajectories for some devices might make implementation difficult.

\section{CONCLUSIONS}

Cursor positioning typically involves prolonged amounts of terminal visual guidance, indicating that enhancements that reduce terminal visual guidance would be effective. Accupoint and Trackball are inefficient cursor control devices, difficult to plan with, and prone to greater overshooting, and with less predictable cursor trajectories. The Mouse and Digitising Pen are more efficient cursor control devices, nevertheless, as detachable manipulanda, there may be problems establishing mappings between device and screen orientation, which are magnified on the screen, and so any interventions should not be engaged for nearer targets for these devices. 


\section{REFERENCES}

Baber, C. (1997). Beyond the desktop. San Diego: Academic Press.

Dix, A.J., Finlay, J.E., Abowd, G.D., \& Beale, R. (1998). Human-computer interaction. London: Prentice Hall.

Douglas, S.A., \& Mithal, A.K. (1997). The ergonomics of computer pointing devices. London: Springer.

English, J.D., \& Andre, A.D. (1999). Posture and internet navigation: An observational study. Proceedings of Silicon Valley Ergonomics Conference \& Exposition, San Jose: San Jose University, 126-135.

Fitts, P.M. (1954). The information capacity of the human motor system in controlling the amplitude of movement. Journal of Experimental Psychology, 47, 381-391.

Fogleman, M. \& Brogmus, G. (1995). Computer mouse use and cumulative trauma disorders of the upper extremities. Ergonomics, 38, 2465-2475.

Gan, K.C. \& Hoffmann, E.R. (1988). Geometrical conditions for ballistic and visually controlled movements. Ergonomics, 31, 829-839.

Goodale, M.A., Jakobson, L.S., \& Servos, P. (1996). The visual pathways mediating perception and prehension. In A.M. Wing, P. Haggard, \& J.R. Flanagan (eds.), Hand and Brain. San Diego: Academic Press, 15-31.

Harms-Ringdahl, K. \& Schüldt, K. (1990). Neck and shoulder load and load-elicited pain in sitting work postures. In M.I. Bullock (ed.), Ergonomics: The physiotherapist in the workplace. Edinburgh: Churchill-Livingstone, 133-147.

Hittle, J.M. (1999). Using alternative input devices and techniques to reduce repetitive strain injury. Proceedings of Silicon Valley Ergonomics Conference \& Exposition, San Jose: San Jose University, 221-223.

Kabbash, P. \& Buxton, W. (1995). The "Prince" technique: Fitts' law and selection using area cursors. CHI'95 Mosaic of Creativity, May 7-11, 273-279.

Langolf, G.D., Chaffin, D.B., \& Foulke, J.A., (1976). An investigation of Fitts' law using a wide range of movement amplitudes. Journal of Motor Behavior, 8, 113-128.

MacKenzie, I.S. (1992). Fitts' law as a research and design tool in human-computer interaction. Human-Computer Interaction, 7, 91-139.

Murata, A. (1995). Improvement of performance by method for predicting targets in pointing by mouse. IEICE Transactions Fundamentals, 78, 1537-1541.

Tränkle, U., \& Deutschmann, D. (1991). Factors influencing speed and precision of cursor positioning using a mouse. Ergonomics, 34, 161-174.

Walker, N., Meyer, D.E., \& Smelcer, J.B. (1993). Spatial and temporal characteristics of rapid cursor-positioning movements with electromechanical mice in human-computer interaction. Human Factors, 35, 431-458.

Wickens, C.D., Gordon, S.E., \& Liu, Y. (1998). Engineering psychology and human performance. New York: Harper Collins. 